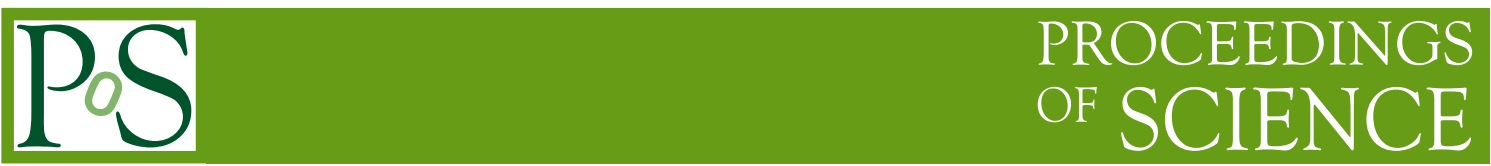

\title{
Unveiling cosmic structure formation with galaxy imaging and redshift surveys
}

\section{Chiaki Hikage*}

Kobayashi-Maskawa Institute for the Origin of Particles and the Universe (KMI),

Nagoya University, 464-8602, Japan

E-mail: hikage@kmi.nagoya-u.ac.jp

Large-scale structure in the Universe provides a key information of various fundamental issues in modern physics: dark energy, absolute mass of neutrinos, the origin of large-scale structure. Different galaxy imaging and spectroscopic surveys including Japan-led "SuMIRe" project using Subaru Telescope is ongoing or planned to unveil the evolution of the large-scale structure at a wide range of redshift. The largest source of systematic uncertainty in cosmological analysis using galaxy dataset is the challenge of modeling the complex relationship between galaxy redshift and the distribution of dark matter. We introduce two novel methods to eliminate this systematics by combining different observables from galaxy spectroscopic and photometric data and also show how the measurement of cosmic growth rate is improved.

KMI International Symposium 2013 on Quest for the Origin of Particles and the Universe",

11-13 December, 2013

Nagoya University, Japan

${ }^{*}$ Speaker. 


\section{Introduction}

Over the past three decades, astronomers have been conducting ever larger redshift surveys in their efforts to probe the large-scale structure of the universe [3, 4, 18, 15]. In the coming decade, we are embarking on even larger surveys including BOSS [19], WiggleZ [2], Subaru PFS [20], Euclid [21]. This upcoming generation of surveys are motivated by our desire to understand cosmic acceleration and to measure the composition of the universe by simultaneously measuring geometry and dynamics. The combination of cosmic microwave background (CMB) data and large redshift surveys trace the growth of structure formation from the last-scattering surface $(z \simeq 1100)$ to low redshifts and determine cosmological parameters to high precision [17]. Measurements of the baryon acoustic oscillation (BAO) scale provide us with a robust geometrical probe of the angular diameter distance and the Hubble expansion rate [5]. Observations of redshift-space distortion measure the growth rate of structure formation [6]. Combining measurements of the growth of structure formation and the geometry of the universe provides a key clue to understanding the nature of dark energy, properties of gravity on cosmological scales, or the nature of cosmic acceleration.

The galaxy power spectrum in redshift space, a direct observable from a redshift survey, is a two-dimensional function of wavelengths perpendicular and parallel to the line-of-sight direction $[15,14,6]$. While galaxy clustering in real space is statistically isotropic in an isotropic and homogeneous universe, the line-of-sight components of galaxies' peculiar velocities alter galaxy clustering in redshift space [12]. The amplitude of the distortion depends both on geometry and dynamics [1].

For the surveys to achieve their ambitious goals for precision cosmology, we will need a detailed understanding of the underlying systematics. One of the major systematic uncertainties in redshift-space power spectrum measurements is non-linear redshift distortion due to the internal motion of galaxies within halos, the so-called Finger-of-God (FoG) effect [11]. Since it is sensitive to highly non-linear physics as well as difficult to model galaxy formation/assembly histories, the FoG effect is the dominant systematic in redshift surveys.

Here we introduce two novel methods to eliminate the systematics due to FoG effect: one is utilizing cross-correlation with photometric galaxies. The other is multipole components of galaxy power spectrum. We find that these techniques improve the measurement of growth rate as well as neutrino mass measurements.

\section{Cross-correlation with photometric galaxies}

Both galaxy-galaxy lensing and cross-correlation with photometric galaxies can be used to infer the amount of the off-centered galaxy contamination and to correct the FoG contamination in the redshift-space power spectrum [7,8]. Cross-correlation of spectroscopic galaxies with background galaxy images, i.e., galaxy-galaxy lensing, and with provides us the information of the matter distribution around the galaxies. As shown in Figure 1, the lensing measurements around the central galaxies exhibit the true profile of the dark matter halos hosting the spectroscopic galaxies. On the other hand, the lensing signals around the off-centered galaxies are diluted at angular scales smaller than the typical offset scale. The plot in Figure 1 demonstrates how the off-centering effect alters the LRG-galaxy weak lensing profile (solid curves) and the projected correlation function of 


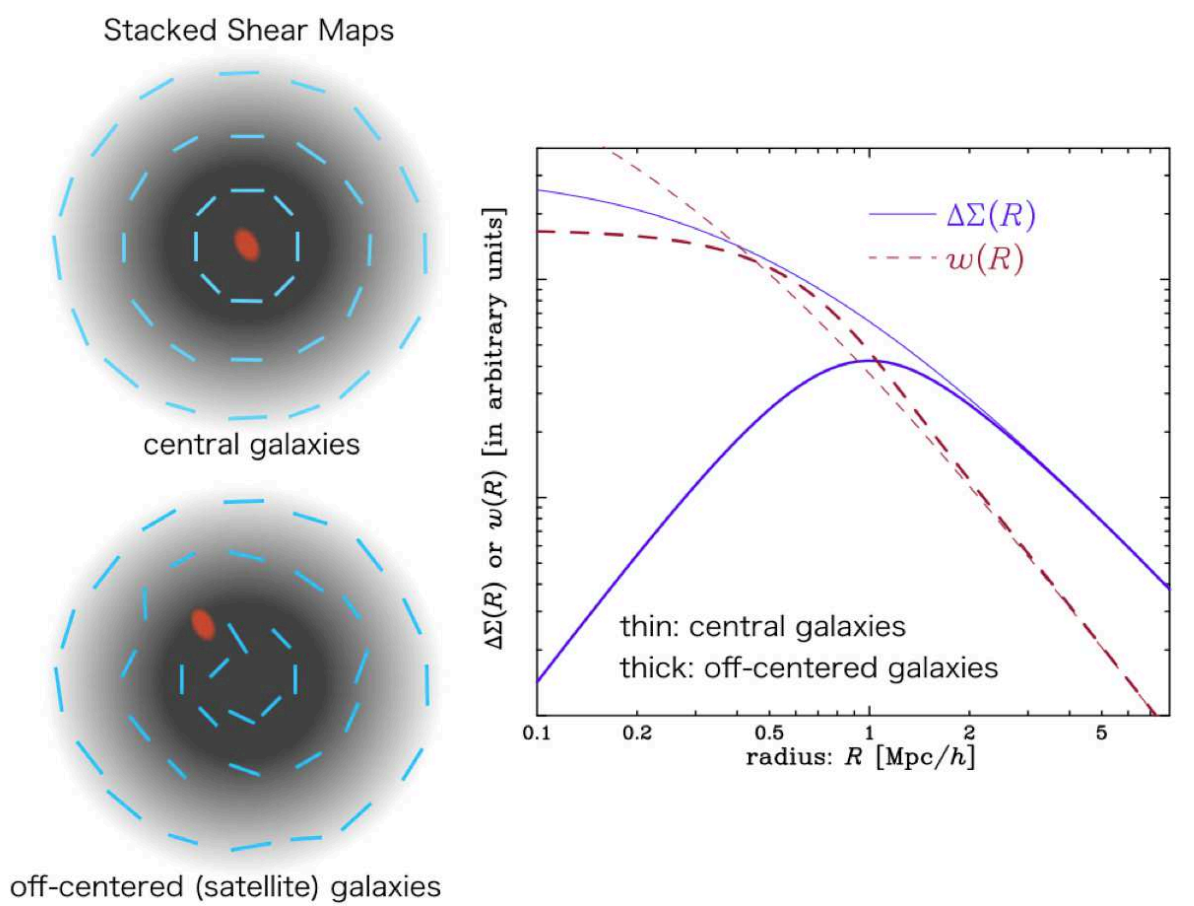

Figure 1: Left figures illustrates the stacked lensing signal around central galaxies (upper) and around offcentered (satellite) galaxies (lower). Lensing signal around the central galaxies reflect the true mass profile of the host halo. On the other hand, the lensing signal is diluted around the off-centered galaxies at the scale smaller than the offset radius. These signature is seen in the galaxy-galaxy lensing $d \Sigma(R)$, and the cross-correlation function with photometric galaxies $w(R)$ where $R$ is the projected radius in the right figure. The thin and thick solid curves show how the off-centering effect dilutes the lensing and cross-correlation profile.

LRGs with photometric galaxy positions (dashed) as a function of the projected radius from the true center or off-centered LRG positions. The amplitude of each profile (in the $y$-axis) is plotted in arbitrary units. We assume $M_{180 b}=1.6 \times 10^{14} M_{\odot} / h$ and $c_{180 b}=4.8$ for the halo mass and concentration parameters, respectively, and employed $R_{\text {off }}=400 \mathrm{kpc} / h$ for the off-centering parameter. The weak lensing distortion probes the gravitational tidal field around lensing halos and the crosscorrelation of LRGs with galaxies probes the projected number density profile of galaxies in the host halos. The off-centering effect dilutes the correlation function amplitudes at radii smaller than the off-centering radius. However, note different features of the off-centering effects on the two profiles. For the projected correlation function, the off-centering effect causes an enhancement in the amplitude over a range of the intermediate radii; $w_{\text {off }}(R)>w_{\mathrm{w} / \mathrm{o} \text { off }}(R)$. On the other hand, the inequality $\Delta \Sigma_{\text {off }}(R) \leq \Delta \Sigma_{\mathrm{w} / \mathrm{o} \text { off }}(R)$ always holds for the LRG-galaxy lensing profile due to the non-local nature of the tidal field.

We use the Sloan Digital Sky Survey (SDSS) DR7 Luminous Red Galaxy catalog, which has been widely used for the cosmological analysis, and measure the off-centered fraction in the catalog. The catalog consists of 96,762 LRGs with absolute magnitude $-23.2<M_{g}<-21.2$ in the redshift range $0.16<z<0.47$ covering about $1.58(\mathrm{Gpc} / h)^{3}$ comoving volume. We use the 

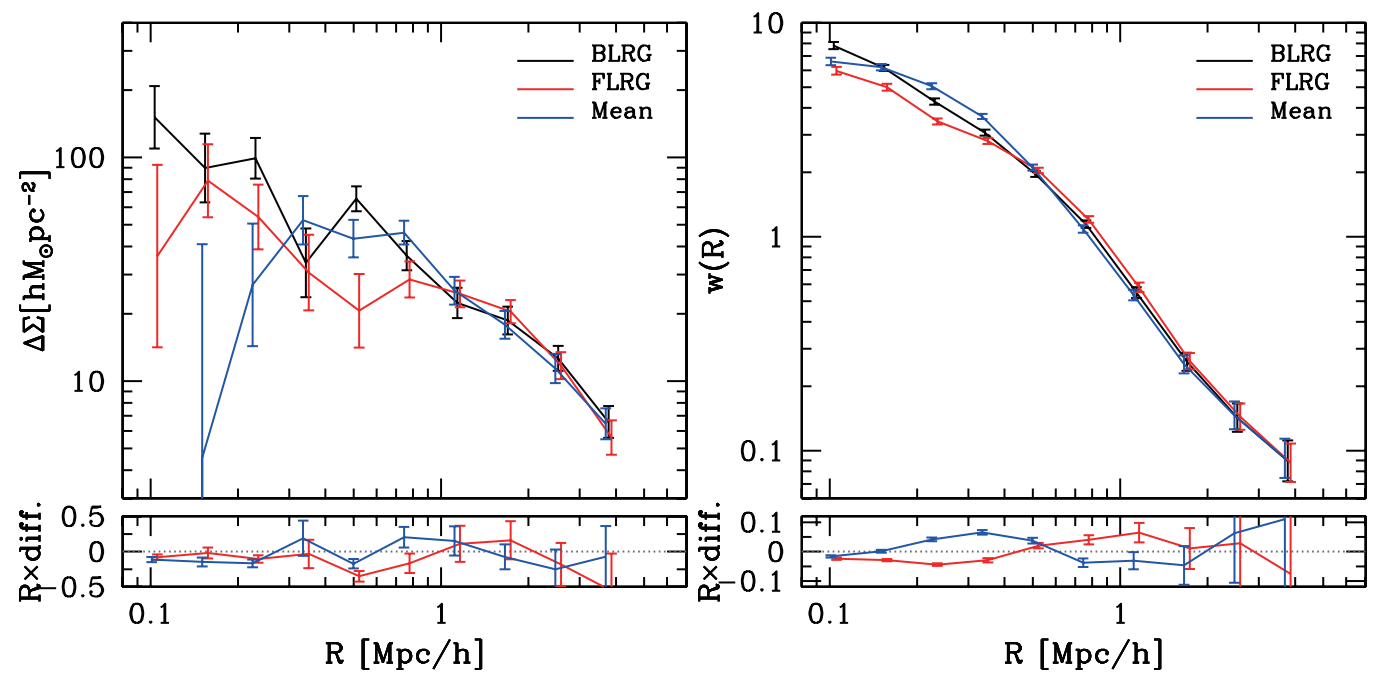

Figure 2: Expectation of FoG effect in SDSS DR7 LRG sample.

Counts-in-Cylinders (CiC) techniques developed by [16] to identify groups of $N_{\mathrm{LRG}} \geq 2$ LRGs occupying the same halo. The total number of halos inferred from the LRG catalog is 92,046, and about 4.5 per cent are multiple-LRG systems. Among the multiple-LRG systems, about 90 per cent are systems with two LRGs $\left(N_{\mathrm{LRG}}=2\right)$. To compute the power spectrum and the crosscorrelations from the LRG-inferred halos, we must define the halo center for each LRG system. (1) For single LRG systems with $N_{\mathrm{LRG}}=1$, we use the redshift and angular position of LRG as the halo center proxy. (2) For multiple-LRG systems with $N_{\mathrm{LRG}} \geq 2$, we use the following three halo center proxies in order to compare the measurements:

- BLRG: the brightest LRG (BLRG) in each multiple-LRG group as the halo center. The BLRG is expected to reside in the most massive sub-halo and therefore be closer to the underlying true center due to dynamical friction theory.

- FLRG: the faintest LRG (FLRG) as the halo center; it can be considered as the extreme counterpart to BLRG.

- Mean: the mean position of LRGs in their redshift and angular positions. Note that we did not use any luminosity or other weighting to estimate the mean position.

Figure 2 shows the LRG-galaxy lensing measurement and the cross-correlation with photometric galaxies, around BLRG, FLRG or Mean halo center proxies, respectively. The three lensing signals at radii $\geq 1 \mathrm{Mpc} / h$ are similar, but the signals at smaller radii differ from each other. The lower panel shows the fractional difference of the profile relative to BLRG center, multiplied by $R$ for illustrative purpose: (FLRG/BLRG -1$) \times R$ or $($ Mean/BLRG -1$) \times R$. The error bars for the ratio properly take into account the cross-covariance of the different profiles, because these different-center measurements are from the same multiple-LRG systems as well as from the same population of background galaxies and therefore the errors of the different profiles are highly correlated with each other at each radial bin. 


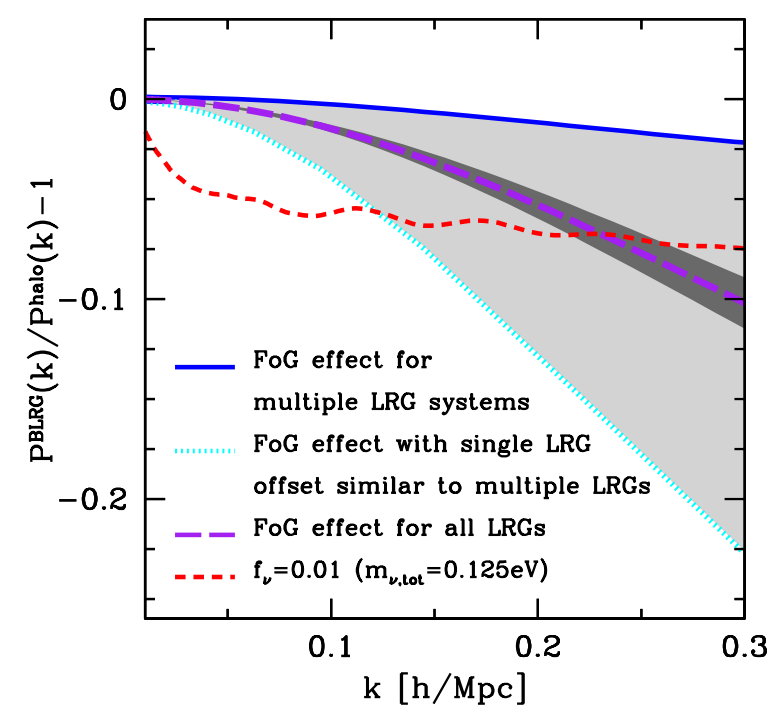

Figure 3: Expected FoG suppression on the monopole power spectra for SDSS DR7 LRG sample using the BLRG center proxy.

Figure 3 shows the expected FoG effect in the SDSS LRG sample. The upper solid curve of the shade region shows the FoG effect assuming that only BLRGs in the multiple-LRG systems have the off-centering effects. The lower dotted curve shows the FoG effect assuming that BLRGs in the single LRG systems also have off-centering effects. The bold dashed curve is the FoG effect implied from the constraints on off-centering parameters for the single-LRG systems, and the dark shaded region around the curve implies the range covered by $1 \sigma$ uncertainties of the off-centering parameters. For comparison, the dashed curve shows the suppression effect caused by massive neutrinos with total mass $f_{v}=0.01\left(m_{v \text {,tot }}=0.125 \mathrm{eV}\right)$. This reveals that the possible FoG effect suggested by the results in this paper can be a serious source of systematic errors in estimating cosmological parameters using the LRG power spectrum without directly modeling the effect.

\section{Multipole component of galaxy power spectra}

We propose another way to correct FoG effect using the multipole components of galaxy power spectra $P_{l}(k)$ generated by the FoG effect. [9] report a clear detection of high- $l$ multipole anisotropy such as hexadecapole $(l=4)$ and tetra-hexadecapole $(l=6)$, which comes from the FoG effect of one-halo term, the contributions of central-satellite and satellite-satellite pair hosted by the same halos. Figure 4 shows the effect of the non-BLRG (NBLRG) galaxies on the hexadecapole component of the galaxy power spectrum $P_{4}(k)$. The black curve is the results with the All LRG sample, the green curve is the one with the Single LRG sample, and the blue curve is the one with the BLRG sample. The difference between the green and blue is small, which means that the difference between the Single LRG sample and the BLRG sample is small. But the difference between the black curve and the blue curve is significant, which means that the contribution from the NBLRG sample is crucial though the fraction of the NBLRGs are small. 

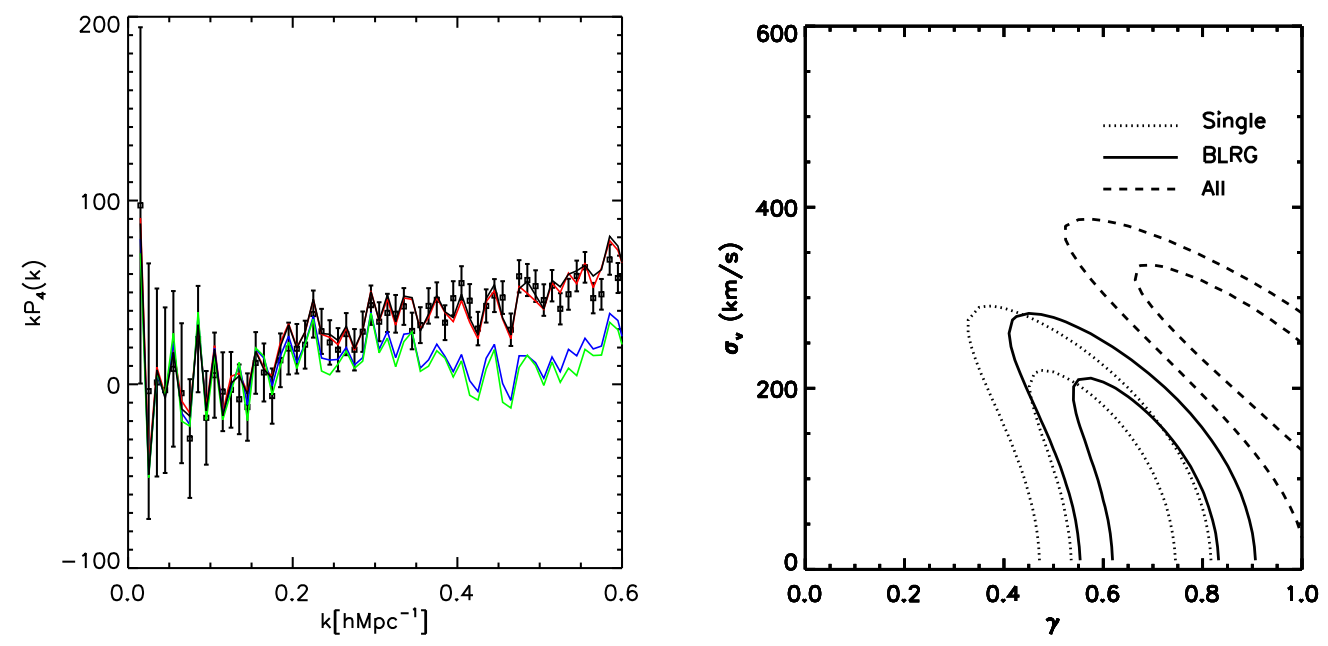

Figure 4: Left: Multipole power spectrum $P_{4}(k)$ for the All LRG sample (black curve), the BLRG sample (blue curve), and the Single LRG sample (green curve). The squares with the small error bars show the results with the sample in a previous paper for comparison. Right: Contour of $\Delta \chi^{2}$ on $\widetilde{\sigma}_{v}$ and $\gamma$ plane. The solid (dotted) curves are the 1 sigma and the 2 sigma contours with the power spectrum with the brightest (single) LRG sample, while the dashed curve is the same but with the All LRG sample. The left panel used the data in the range of wavenumbers $0.01 \mathrm{hMpc}^{-1} \leq k \leq 0.2 \mathrm{hMpc}^{-1}$, but the right panel used the data in the range $0.01 h \mathrm{Mpc}^{-1} \leq k \leq 0.3 h \mathrm{Mpc}^{-1}$.

Right panel in Figure 4 also shows the 1 sigma and 2 sigma contours of $\Delta \chi^{2}$ on the parameter plane of velocity dispersion $\widetilde{\sigma}_{v}$ and the growth rate index $\gamma, f_{z}=\frac{d \log D(z)}{d \log a}=\Omega_{m}(a)^{\gamma}$, where $\Omega_{m}(a)$ is the matter density parameter at the scale factor $a$. Here we fixed the other parameters $n_{s}=0.97$, $\Omega_{m}=0.28, \Omega_{b}=0.046, \sigma_{8}=0.8$ and assumed the cold dark matter model with a cosmological constant as the background universe model. In each panel, the dotted curve, solid curve, and the dashed curve are the Single, Brightest, and All LRG sample, respectively. The left (right) panel used the data with $k \leq 0.2 \mathrm{hMpc}^{-1}\left(k \leq 0.3 \mathrm{hMpc}^{-1}\right)$. The value $\gamma=0.55$ is the prediction of the model on the basis of the general relativity. Though our theoretical model is very simple, the results clearly show that the contamination of the satellite galaxies (NBLRGs) significantly biases the parameter estimation. This figure also indicates that the results are influenced by including the brightest LRGs consisting of the multiple systems.

As the off-centered or satellite galaxies have large internal motion, the FoG effect provides a useful probe of constraining the fraction of satellite galaxies. Figure 5 shows the joint constraints on the fraction of satellite galaxies $f_{\text {sat }}$ and the growth rate index $\gamma$ and the satellite velocity dispersion $\sigma_{v}^{\text {(sat) }}$ from different combinations of $P_{l}$. The parameters $f_{\text {sat }}$ and $f_{z}$ degenerate with each other because the suppression of quadrupole power due to satellite FoG effect is mimicked by increasing the growth rate $f_{z}$ (or decreasing $\gamma$ ). Figure 5 shows that the addition of high- $l$ multipole measurements breaks their degeneracy and then improves the accuracy of $\gamma$ by nearly twice. The input values denoted by the cross symbols are successfully reproduced [10]. 

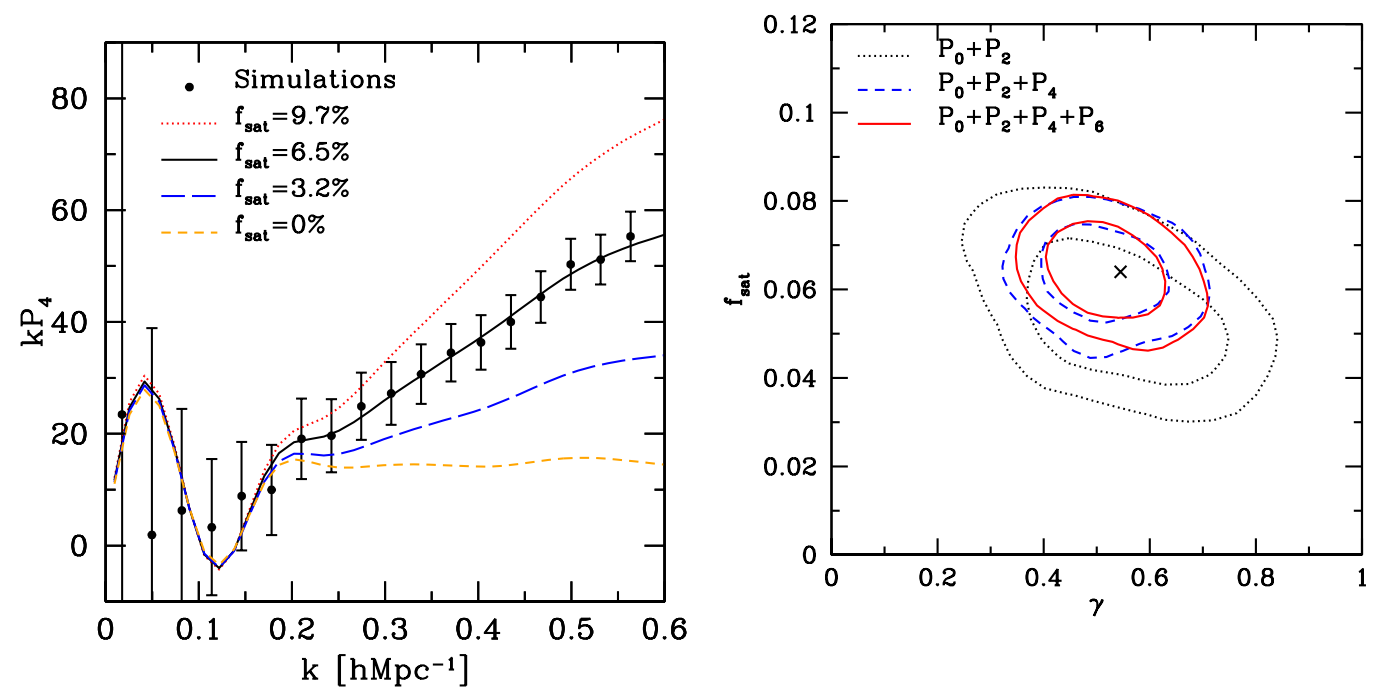

Figure 5: Left: Hexadecapole $P_{4}(k)$ for the mock LRG samples (black circles) with 1- $\sigma$ error-bars. The black lines represent the model prediction with the fiducial values of HOD in which the satellite fraction is $f_{\text {sat }}=6.5 \%$ and the satellite velocity dispersion is $\sigma_{v}^{(\text {sat })}=570 \mathrm{~km} / \mathrm{s}$. For comparison, we plot the theoretical models by varying $f_{\text {sat }}$. Right: Joint constraints on the satellite fraction $f_{\text {sat }}$ and the growth rate index $\gamma$ (left) or $\sigma_{v}^{\text {(sat) }}$ (right) from $P_{0}+P_{2}$ (black), $P_{0}+P_{2}+P_{4}$ (blue), and $P_{0}+P_{2}+P_{4}+P_{6}$ (red). Here we use a functional form of HOD obtained by [16]. Each contour denotes $68 \%$ and $95 \%$ error respectively. The symbol of crosses denote the input values.

\section{Summary}

Goals of current and upcoming galaxy surveys are testing general relativity at cosmological scale and measuring the absolute neutrino mass. Major challenge in this attempt comes from the uncertainty in the relationship between galaxy distribution and underlying dark matter distribution. We use the lensing measurements to determine the amplitude of this effect and to determine the expected amplitude of FoG effects. We develop an approach for using the lensing data to model how the FoG suppresses the power spectrum amplitudes and show that the current data implies a $30 \%$ suppression at wavenumber $k=0.2 \mathrm{hMpc}^{-1}$. Joint imaging and spectroscopic surveys allow a robust, unbiased use of the power spectrum amplitude information: it improves the marginalized error of growth rate $f_{g} \equiv d \ln D / d \ln a$ by up to a factor of 2 over a wide range of redshifts $z<1.4$. We also find that the dark energy equation-of-state parameter, $w_{0}$, and the neutrino mass, $f_{v}$, can be unbiasedly constrained by combining the lensing information, with an improvement of 10-25\% compared to a spectroscopic survey without lensing calibration [7].

The high- $l$ multipole spectra such as $P_{4}$ and $P_{6}$ are sensitive to the Fingers-of-God (FoG) effect due to the large internal motion of satellite galaxies and thus they are useful probe to constrain the fraction and the velocity dispersion of satellite galaxies. We find that the input HOD is successfully reconstructed from $P_{l}$ and that high- $l$ multipole spectra significantly improve the accuracy of the HOD measurements. We also find that the addition of high- $l$ multipole information improve the accuracy of the growth rate measurement by nearly twice because they break the degeneracy between the cosmic growth rate and the satellite FoG. 


\section{References}

[1] C. Alcock, B. Paczynski, Nature 281 (1979) 358

[2] Blake C., et al., Mon. Not. Roy. Astron. Soc. 415 (2011) 2876 [arXiv:1104.2948]

[3] Davis M., Huchra J., Astrophys. J. 254 (1982) 437

[4] de Lapparent V., Geller M. J., Huchra J. P., Astrophys. J. Lett. 302 (1986) L1

[5] Eisenstein D. J., et al., Astrophys. J.633 (2005) 560 [arXiv:0501171]

[6] Guzzo L., et al., Nature 451 (2008) 541 [arXiv:0802.1944]

[7] C. Hikage, M. Takada and D. N. Spergel, Mon. Not. Roy. Astron. Soc. 419 (2012) 3457 [arXiv:1106.1640]

[8] C. Hikage, R. Mandelbaum, M. Takada, and D. N. Spergel, Mon. Not. Roy. Astron. Soc. 435 (2013) 2345 [arXiv:1211.1009]

[9] C. Hikage, K. Yamamoto, J. Cosmol. Astropart. Phys. 8 (2013) 19 [arXiv:1303.3380]

[10] C. Hikage, Mon. Not. Roy. Astron. Soc. Lett.in press [arXiv:1401.1246]

[11] Jackson J. C., Mon. Not. Roy. Astron. Soc. 156 (1972) 1P

[12] Kaiser N., Mon. Not. Roy. Astron. Soc. 227 (1987) 1

[13] S. Masaki, C. Hikage, M, Takada, D. N. Spergel, and N. Sugiyama, Mon. Not. Roy. Astron. Soc. 433 (2013) 3506 [arXiv:1311.7077]

[14] T. Okumura, T. Matsubara, D. J. Eisenstein, I. Kayo, C. Hikage, A. S. Szalay, D. P. Schneider, Astrophys. J. 676 (2008) 889 [arXiv:0711.3640]

[15] Peacock J. A., et al., Nature 410 (2001) 169 [arXiv:astro-ph/0103143]

[16] Reid B. A., Spergel D. N., Astrophys. J. 698 (2009) 143 [arXiv:0809.4505]

[17] Wang Y., Spergel D. N., Strauss M. A., Astrophys. J. 510 (1999) 20 [arXiv:astro-ph/9802231]

[18] York D. G., et al., Astron. J. 120 (2000) 1579 [arXiv:astro-ph/0006396]

[19] K. S. Dawson, et al. Astron. J. 145 (2013) 10 [arXiv:1208.0022]

[20] Takada M., et al., Pub. Astron. Soc. Japan 66 (2014) 66 [arXiv:1206.0737]

[21] R. Laureijs, et al. (2011) [arXiv:1110.3193] 